\title{
Mitochondrial DNA T7719G in tRNA-Lys gene affects litter size in Small-tailed Han sheep
}

\author{
Xiaoyong Chen ${ }^{1,2 \dagger}$, Dan Wang ${ }^{1 \dagger}$, Hai Xiang ${ }^{1}$, Weitao Dun², Dave O. H. Brahi ${ }^{1}$, Tao Yin ${ }^{1}$ and Xingbo Zhao ${ }^{1 *}$
}

\begin{abstract}
Background: In farm animals, mitochondrial DNA (mtDNA) effect on economic performance remains hot-topic for breeding and genetic selection. Here, 53 maternal lineages of Small-tailed Han sheep were used to investigate the association of mitochondrial DNA variations and the lambing litter size.

Results: Sequence sweeping of the mitochondrial coding regions discovered 31 non-synonymous mutations, and the association study revealed that T7719G in mtDNA tRNA-Lys gene was associated with litter size $(P<0.05)$, manifesting 0.29 lambs per litter between the $G$ and T carriers. Furthermore, using the mixed linear model, we assayed the potential association of the ovine litter size and haplogroups and multiple-level mtDNA haplotypes, including general haplotypes, assembled haplotypes of electron transport chain contained sequences (H-ETC), mitochondrial respiratory complex contained sequences (H-MRC) and mitochondrial genes ( $\mathrm{H}$-gene, including polypeptide-coding genes, rRNA genes and tRNA genes). The strategy for assembled mitochondrial haplotypes was proposed for the first time in mtDNA association analyses on economic traits, although none of the significant relations could be concluded $(P>0.05)$. In addition, the nuclear major gene BMPR1B was significantly correlated with litter size in the flock $(P<0.05)$, however, did not interact with mtDNA T7719G mutation $(P>0.05)$.
\end{abstract}

Conclusions: Our results highlight mutations of ovine mitochondrial coding genes, suggesting T7719G in tRNA-Lys gene be a potentially useful marker for selection of sheep litter size.

Keywords: Association, Haplotype, Mitochondria, Non-synonymous mutation, Reproduction, Sheep

\section{Background}

Mitochondria are responsible for ATP production in the electron transport chain (ETC) in cells. The ETC consists of five mitochondrial respiratory complexes (MRCs), of which complex I, complex III, complex IV and complex $\mathrm{V}$ are constructed by both mitochondrial and nuclear encoded proteins, and complex II is entirely encoded by nuclear genes. Mitochondrial genome codes 13 polypeptides, 2 rRNAs and 22 tRNAs [1]. Since the first report of mitochondrial DNA (mtDNA) effect on milk production traits in dairy cattle [2], mtDNA effects on various economic traits have been widely studied in livestock, including pigs $[3,4]$, dairy cattle $[5,6]$, beef

\footnotetext{
* Correspondence: zhxb@cau.edu.cn

${ }^{\dagger}$ Equal contributors

${ }^{1}$ College of Animal Science and Technology, China Agricultural University,

Beijing 100193, China

Full list of author information is available at the end of the article
}

cattle $[7,8]$, sheep $[9,10]$ and chickens $[11,12]$. Litter size is one of the vital economic traits for animal breeding and production, which has been studied for decades. For sheep, the nuclear gene, BMPR1B was identified as one of causative genes for sheep prolificacy [13], and has been widely used in sheep breeding. For the mtDNA effect on litter size, researchers reported the association with ewe litter size among haplogroups in an Afec-Assaf flock, but did not found the interaction with $B M P R 1 B$ effect [10]. However, previous studies reported poor mtDNA effects [14-17], which made it necessary to uncover the genetic contribution of mtDNA for ewe litter size. In this study, Small-tailed Han sheep, a prolific breed of China, were used to investigate the association of litter size with mtDNA coding genes, in which nonsynonymous mutations were considered as possible functional SNPs. Besides the non-synonymous mutation and haplogroup, assembled haplotypes of ETC- 
contained mtDNA sequences, MRC-contained mtDNA sequences, and mitochondrial genes were analyzed, respectively. The strategy for assembled mitochondrial haplotypes was proposed for the first time in association analyses. In addition, BMPR $1 B$ effect and interaction with mtDNA mutations were analyzed.

\section{Methods}

\section{Animals}

In total, 117 lambing Small-tailed Han sheep from 53 maternal lineages (families divided by female ancestors) of the same flock were performed blood sample collection, and recorded one or more times of litter size (Additional file 1: Table S1). All sheep were kept indoors year-round, and fed with mixed silage and hay to meet their nutritional requirements. Litter size (number of lambs born) was recorded, and full pedigree information was collected for all animals as reproductive management of the flock where ewes were mated with the selected rams after spontaneous estrus. All the ewes were genotyped for the BMPR1B at >2-month age.

\section{Genotyping of BMPR1B and sequencing of mitochondrial coding genes}

Genomic DNA was extracted using the standard phenol/ chloroform method [18]. The BMPR1B was genotyped using PCR-RFLP assay with the Ava II restriction enzyme [19]. Mitochondrial complete coding sequences were amplified by 17 primer pairs [20], and PCR products were sequenced in the Sanger method.

\section{Haplotype and haplogroup constitution}

All non-synonymous mutations were used to constitute the haplotype and haplogroup. Considering the possible action for mitochondrial function, haplotypes were furthermore assembled by mtDNA sequence of ETC, MRCs and genes respectively. Haplotypes were determined by the online software FaBox [21], and haplogroups were constituted based on network analysis by Network 4.6.1.4 [22].

\section{Association analysis}

Association analyses were carried out in the following mixed model by MIXED procedure in SAS software version 9.2 (SAS Institute Inc., Cary, North Carolina, USA).

$$
\begin{aligned}
l s= & y s+\text { parity }+ \text { ram }+B M P R 1 B+\text { mutations } \\
& +B M P R 1 B \times \text { mutations }+I D+E P+e
\end{aligned}
$$

In the model, the effects of lambing year-season $(y s)$, parity number (parity), service ram (ram), BMPR1B genotype $(B M P R 1 B)$, mtDNA mutations (mutations, including the effects of mtDNA non-synonymous mutations, haplotypes and haplogroups), the interaction between BMPR1B and mtDNA mutations $(B M P R 1 B \times$ mutations), the polygenic effect (ID), the permanent environmental effect $(E P)$, and the random residual $(e)$ were included. The response variable was the ewe litter size $(l s)$. Each cell of these effects contained observations. The polygenic effect corrected the genetic background by the additive genetic relationship matrix, i.e. the pedigree information. The permanent environmental effect dealed with the repeated measurement data.

Table 1 Non-synonymous mutations in mitochondrial coding

\begin{tabular}{|c|c|c|c|c|}
\hline Gene & Nucleotide $^{a}$ & $\begin{array}{l}\text { Codon } \\
\text { mutation }\end{array}$ & $\begin{array}{l}\text { Amid acid } \\
\text { substitution }\end{array}$ & Significance \\
\hline ND1 & T3543A & $\mathrm{UCA} \rightarrow \mathrm{ACA}$ & $S \rightarrow T$ & ns \\
\hline ND2 & T4208C & $\mathrm{A} \cup \mathrm{A} \rightarrow \mathrm{ACA}$ & $M \rightarrow T$ & ns \\
\hline COII & C7500A & $\mathrm{CCC} \rightarrow \mathrm{CAC}$ & $\mathrm{P} \rightarrow \mathrm{H}$ & ns \\
\hline \multirow[t]{2}{*}{ ATP6 } & A8039G & $\mathrm{AAC} \rightarrow \mathrm{AGC}$ & $\mathrm{N} \rightarrow \mathrm{S}$ & ns \\
\hline & G8264C & $\mathrm{GGA} \rightarrow \mathrm{GCA}$ & $\mathrm{G} \rightarrow \mathrm{A}$ & ns \\
\hline COIII & A9375G & $\mathrm{A} \cup \mathrm{A} \rightarrow \mathrm{G} \cup \mathrm{A}$ & $M \rightarrow V$ & ns \\
\hline \multirow[t]{2}{*}{ ND4L } & C9974T & $\mathrm{CCU} \rightarrow \mathrm{UCU}$ & $P \rightarrow S$ & ns \\
\hline & G10118A & $\mathrm{GGU} \rightarrow \mathrm{AGU}$ & $\mathrm{G} \rightarrow \mathrm{S}$ & ns \\
\hline \multirow[t]{2}{*}{ ND4 } & G10937A & $\mathrm{GAC} \rightarrow \mathrm{AAC}$ & $\mathrm{D} \rightarrow \mathrm{N}$ & ns \\
\hline & G11045A & $\mathrm{GUU} \rightarrow \mathrm{AUU}$ & $V \rightarrow I$ & ns \\
\hline \multirow[t]{2}{*}{ ND5 } & G12571C & $\mathrm{GGC} \rightarrow \mathrm{GCC}$ & $\mathrm{G} \rightarrow \mathrm{A}$ & ns \\
\hline & G13041A & $\mathrm{GCA} \rightarrow \mathrm{ACA}$ & $\mathrm{A} \rightarrow \mathrm{T}$ & ns \\
\hline \multirow[t]{7}{*}{ ND6 } & C13576T & $\mathrm{CUC} \rightarrow \cup \cup \mathrm{U}$ & $\mathrm{L} \rightarrow \mathrm{F}$ & ns \\
\hline & T13588C & $\mathrm{UAC} \rightarrow \mathrm{CAC}$ & $\mathrm{Y} \rightarrow \mathrm{H}$ & ns \\
\hline & C13777T & $\mathrm{CAU} \rightarrow \mathrm{UAU}$ & $\mathrm{H} \rightarrow \mathrm{Y}$ & ns \\
\hline & C13789T & $\mathrm{CAU} \rightarrow \mathrm{UAU}$ & $H \rightarrow Y$ & ns \\
\hline & T13837C & $\mathrm{UCA} \rightarrow \mathrm{CCA}$ & $S \rightarrow P$ & ns \\
\hline & T13855C & $\cup \cup C \rightarrow C \cup C$ & $F \rightarrow L$ & ns \\
\hline & A13876G & $\mathrm{A} \cup \mathrm{A} \rightarrow \mathrm{G} \cup \mathrm{A}$ & $M \rightarrow V$ & ns \\
\hline \multirow[t]{3}{*}{ 12SrRNA } & T281C & - & - & ns \\
\hline & C291T & - & - & ns \\
\hline & A538G & - & - & ns \\
\hline \multirow[t]{5}{*}{ 16SrRNA } & A1099T & - & - & ns \\
\hline & $\mathrm{T} 1112 \mathrm{C}$ & - & - & ns \\
\hline & T2199A & - & - & ns \\
\hline & C2443T & - & - & ns \\
\hline & T2634C & - & - & ns \\
\hline tRNA-Tyr & G5295A & - & - & ns \\
\hline tRNA-Lys & T7719G & - & - & * \\
\hline tRNA-His & C11606T & - & - & ns \\
\hline tRNA-Ser & G11668A & - & - & ns \\
\hline
\end{tabular}
genes and corresponding effects on litter size

${ }^{a}$ Mutation positions were defined according to the ovine mitochondrial sequence (GenBank Accession nos.: AF010406)

bWen a set of statistical inferences were simultaneously considered, multiple comparisons were conducted by the FDR using the R project. " $\mathrm{ns}$ " represents "not significant", and "*" represents "significant" at the significant level of 0.05 
Inference about the interaction effect was made. If non-significant, that effect was dropped from the model and inference was made about the main effects. If significant, the cell means for the interaction became of interest.

When a set of statistical inferences were simultaneously considered, multiple comparisons were conducted by the false discovery rate (FDR) in the R project ( $\mathrm{R}$ version 3.2.5) [23].

\section{Results}

\section{Mutations in mitochondrial coding genes}

In total, 95 mutations in mitochondrial coding genes were discovered (Additional file 1: Table S2), including 64 synonymous and 31 non-synonymous mutations (19 missense mutations in protein coding genes, 8 mutations in rRNAs, and 4 mutations in tRNAs respectively), which were illustrated in Table 1 .

\section{BMPR1B genotypes}

For $B M P R 1 B$ gene, 117 ewes were genotyped, including ++ genotype of 18 ewes, $\mathrm{B}+$ genotype of 87 ewes and $\mathrm{BB}$ genotype of 12 ewes.

\section{Effects of mitochondrial haplotype and haplogroup on ovine litter size}

The 31 mitochondrial non-synonymous mutations assigned the Small-tailed Han sheep flock to 44 haplotypes (Additional file 1: Table S3), which were clustered into 4 haplogroups (Fig. 1). Using the mixed linear model, no significant effect on any haplotype or haplogroup was associated with litter size of Small-tailed Han sheep $(P>$ $0.05)$, and the interaction between $B M P R 1 B$ and haplotype or haplogroup also did not significantly affect litter size
$(P>0.05)$, while the $B M P R 1 B$ was positively associated with litter size $(P<0.05)$.

\section{Effects of missense mutations and haplotypes in mitochondrial protein coding genes on ovine litter size} Totally $35 \mathrm{H}$-ETCs were constituted by 19 missense mutations in protein coding sequences, while 15 missense mutations in MRCI assembled $33 \mathrm{H}$-MRCIs, and both 2 mutations in MRCIV and MRCV constituted $3 \mathrm{H}$-MRCIVs and $3 \mathrm{H}$-MRCVs respectively. Intensively, $\mathrm{H}$-genes were assembled by mutations of individual genes (Table 2 and Additional file 1: Table S4, S5 and S6). With mixed linear model analyses, no significant association was detected between litter size and the 19 non-synonymous mutations $(P>0.05)$ (Table 1$)$, nor was any haplotype $(P>0.05)$ (Table 2). BMPR1B was strongly associated with litter size $(P<0.05)$ (Table 3$)$, but the interaction of BMPR1B and mtDNA mutations was not remarkable $(P>0.05)$.

\section{Effects of mutations and haplotypes in mitochondrial rRNA genes on ovine litter size}

The 3 mutations in 12SrRNA sorted the sheep flock into 4 haplotypes (H-12SrRNA), and the 5 mutations in $16 \operatorname{SrRNA}$ constituted 15 haplotypes (H-16SrRNA) (Table 2 and Additional file 1: Table S7). Association analyses revealed that neither mutation nor haplotype in rRNA genes affected litter size $(P>0.05)$ (Tables 1 and 2$)$. The BMPR1B genotype was remarkably associated with litter size $(P<0.05)$ (Table 3), however, was inconspicuously correlated to rRNA mutations $(P>0.05)$.

\section{Effects of mutations in mitochondrial tRNA genes on} ovine litter size

There were 4 mutations in tRNA genes, and only one variation was observed in each of them (Table 1),

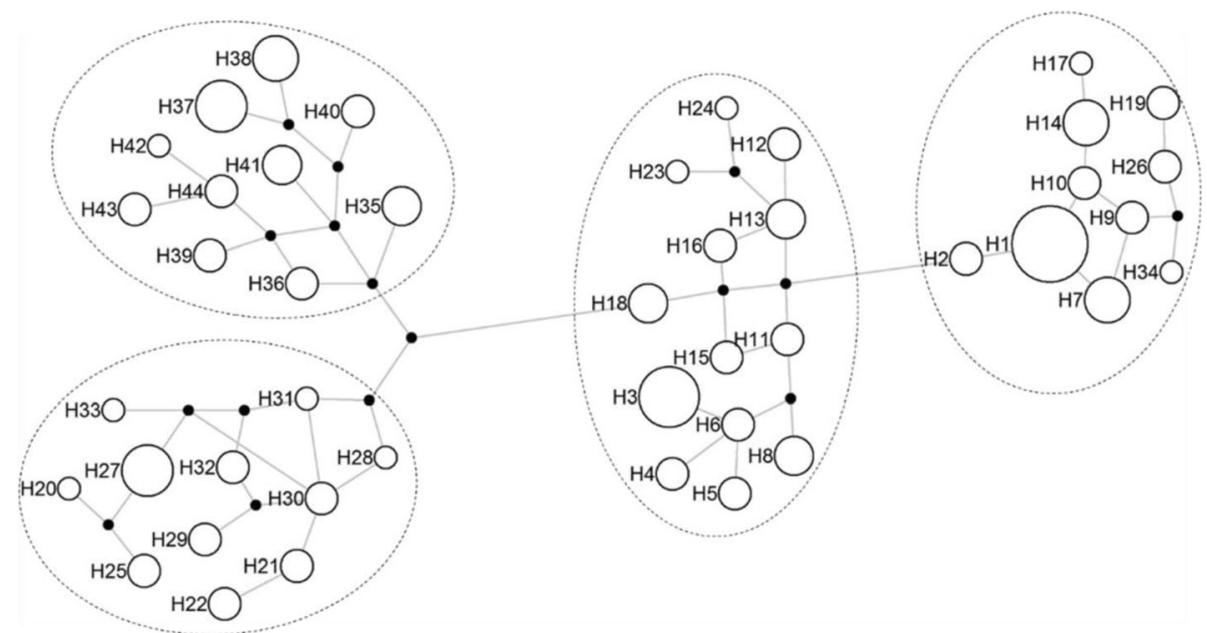

Fig. 1 Neighbor-joining tree of different maternal pedigrees for haplogroup assignments by the 31 non-synonymous mutations 
Table 2 Haplotypes constituted by mitochondrial non-synonymous mutations in multiple levels and significant effects on litter size

\begin{tabular}{|c|c|c|c|c|}
\hline Functional haplotype $^{a}$ & Contained gene & Contained mutation number & Haplotype number & Significance \\
\hline $\mathrm{H}$ & $\begin{array}{l}\text { ND1, ND2, ND4L, ND4, ND5, ND6, COII, COIII, ATP6, } \\
\text { 12SrRNA, 16SrRNA, tRNA-Tyr, tRNA-Lys, tRNA-His, tRNA-Ser }\end{array}$ & 31 & 44 & ns \\
\hline H-ETC & ND1, ND2, ND4L, ND4, ND5, ND6, COII, COIII, ATP6 & 19 & 35 & ns \\
\hline $\mathrm{H}-\mathrm{MRCl}$ & ND1, ND2, ND4L, ND4, ND5, ND6 & 15 & 33 & ns \\
\hline H-MRCIV & COII, COIII & 2 & 3 & ns \\
\hline H-MRCV & ATP6 & 2 & 3 & ns \\
\hline H-ND4L & ND4L & 2 & 4 & ns \\
\hline $\mathrm{H}-\mathrm{ND} 4$ & ND4 & 2 & 4 & ns \\
\hline H-ND5 & ND5 & 2 & 3 & ns \\
\hline H-ND6 & ND6 & 7 & 10 & ns \\
\hline H-ATP6 & ATP6 & 2 & 3 & ns \\
\hline $\mathrm{H}-12 \operatorname{SrRNA}$ & 12SrRNA & 3 & 4 & ns \\
\hline H-16SrRNA & 16SrRNA & 5 & 15 & ns \\
\hline
\end{tabular}

${ }^{a}$ The general haplotype $(\mathrm{H})$ was assembled by all non-synonymous mutations to reflect the integrated characteristics of mtDNA coding regions. Subsequently, the ETC based haplotype (H-ETC) represented the general feature of all ETC-contained mtDNA sequences. The MRC based haplotype (H-MRC) inferred the integrated signal of particular MRC-contained mtDNA sequences. Here, H-MRC included three types, i.e. MRC-I, MRC-IV and MRC-V. The last was the gene-level haplotype (H-gene), which indicated the information of a particular gene-contained mtDNA sequence

bWhen a set of statistical inferences were simultaneously considered, multiple comparisons were conducted by the FDR using the R project. "ns" represents "not significant", and "*" represents "significant" at the significant level of 0.05

therefore no haplotype was constituted. Notably, T7719G in $t R N A-L y s$ affected litter size by 0.29 lambs per litter between the $\mathrm{G}(1.79)$ and $\mathrm{T}(1.50)$ carriers $(P<$ $0.05)$. Even though the $B M P R 1 B$ was in prominent correlation with litter size $(P<0.05)$ (Table 3$)$, there was no interaction with T7719G $(P>0.05)$ (Additional file 1 : Table S8).

\section{Discussion}

In human diseases, mutations in mitochondrial coding genes led to changes of oxidative phosphorylation enzyme complexes [24]. It is rational to image the possible effects of mtDNA on livestock traits. In this study, the Small-tailed Han sheep were used to explore the correlation of mtDNA and ewe litter size.

In order to investigate the overall mtDNA effects, a novel strategy of assembling mitochondrial haplotypes based on biology functions is proposed. Extensively, there are four levels of mitochondrial haplotypes for mitochondrial biological functions. The general haplotype is assembled by all non-synonymous mutations to reflect the integrated characteristics of mtDNA coding

Table 3 Effects of BMPR1B on litter size

\begin{tabular}{lllc}
\hline Genotype & Ewe number & Parity number & Litter size (Means) $^{1}$ \\
\hline++ & 18 & 69 & $1.4638^{\mathrm{a}}$ \\
$\mathrm{B}+$ & 87 & 285 & $1.7474^{\mathrm{b}}$ \\
$\mathrm{BB}$ & 12 & 47 & $2.1915^{\mathrm{c}}$
\end{tabular}

"Means" represents the arithmetic average on litter size of sheep with the genotype. The FDR method was used to conduct multiple comparisons, and means with different lowercase letters are different at the significant level of 0.05 regions. Subsequently, the ETC based haplotype (H-ETC) represents the general feature of all ETC-contained mtDNA sequences. The MRC based haplotype infers the integrated signal of particular MRC-contained mtDNA sequences. H-MRC includes four types, i.e. MRC-I, MRCIII, MRC-IV and MRC-V. The last is the gene level, which indicates the information of a particular gene-contained mtDNA sequence.

In the mixed linear model, unnecessary factors interfere with the estimation of interested factors, as the degree of freedom is wasted. Therefore, based on the thoughts of multiple models [10] and variable selection in a mixed linear model [25], a two-step method of environmental variable selection was put forward in the association analyses, in which nonsignificant environmental factors were ignored to improve accuracy of genetic estimation. In this study, the environmental factors included lambing month, parity of dam, and service ram, and the genetic factors, which we were interested in, included $B M P R 1 B$ genotype, mtDNA mutations and their interaction. We started a full model (Model I) with all the environmental and genetic factors to test if environmental factors were noises. Results revealed that the effects of parity of dam and service ram were not significant on litter size. Subsequently, these two factors were excluded in the aims to construct an optimized model (Model II), which was used to test the significance of genetic factors, especially the effects of mtDNA mutations on litter size.

Many mitochondrial tRNA mutations in human were reported to be associated with wide range of pathological 


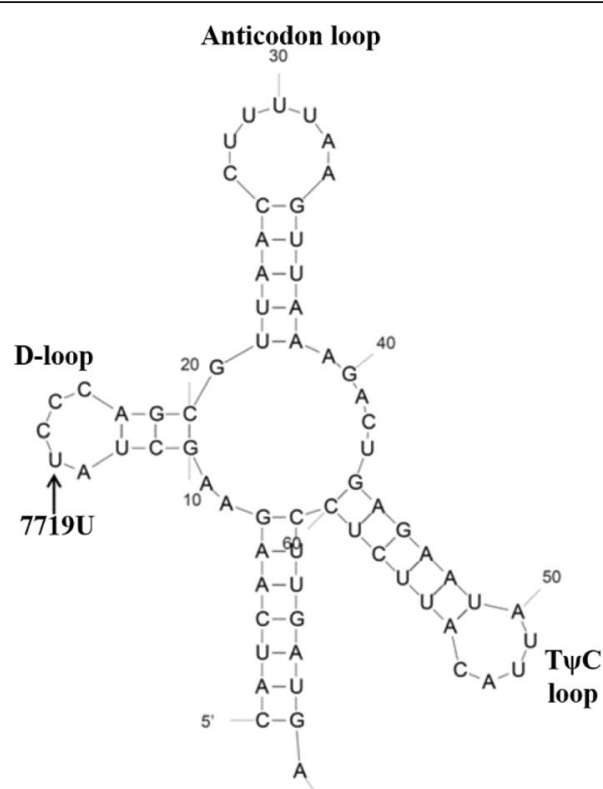

Amino acid acceptor stem

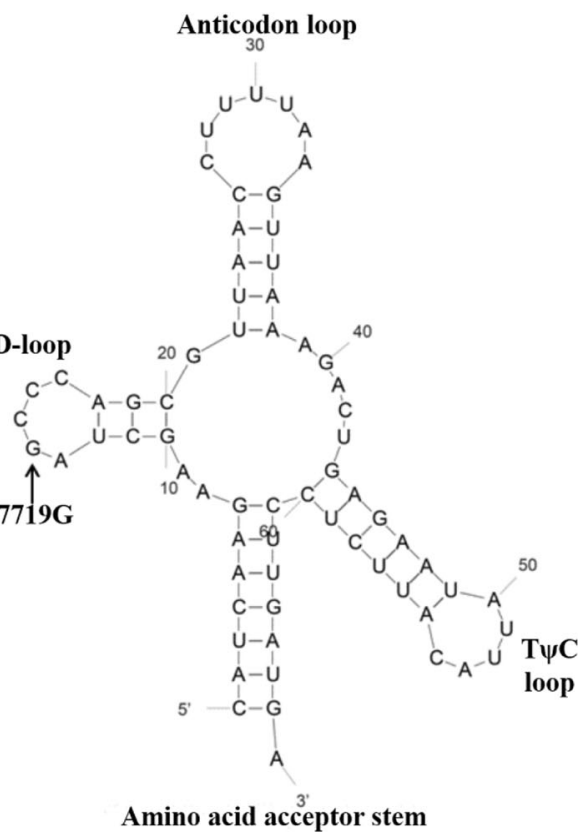

Amino acid acceptor stem

Fig. 2 The predicted secondary structures of the tRNA-Lys for U7719G mutation

conditions [26], for example, deafness was correlated with mitochondrial tRNA-Asp A7551G mutation [27], hypertension was associated with mitochondrial $t R N A$ Ile A4263G mutation [28], and mitochondrial tRNA mutations might decrease in carcinoma hepatocyte [29]. In sheep, A7755G in tRNA-Lys was reported to affect litter size in an Afec-Assaf flock [10], while our study revealed that T7719G in tRNA-Lys was significantly associated with ewe litter size in Small-tailed Han sheep, with the means of 1.79 and 1.50 for the $\mathrm{G}$ and $\mathrm{T}$ carriers, manifesting a difference of 0.29 lambs per litter. Furthermore, the mutation was predicted to produce a transversion of $\mathrm{U}$ to $\mathrm{G}$ at DHU loop on 2D cloverleaf of the tRNA-Lys structure (Fig. 2).

\section{Conclusions}

As a conclusion, we summarize the research procedures for mtDNA effects. Firstly, sweeping mitochondrial variations on maternal lineages; secondly, constituting the haplotype, haplogroup and assembled haplotypes; lastly, analyzing the association between mtDNA mutations (individual mutations, haplotypes and haplogroups) and interested traits. The present study discovered the mtDNA T7719G was linked with ewe litter size. For traits of low heritability, the marker assisted selection could increase the accuracy of breeding and selection. For the further study, the mtDNA T7719G should be put into post-association validation, and may become a genetic marker in the sheep breeding programs.

\section{Additional file}

Additional file 1: Table S1. Detail information of used sheep. Table S2. Information of variations in mtDNA coding region of Small-tailed Han sheep. Table S3. The haplotype constituted by the 31 mitochondrial nonsynonymous mutations in mitochondrial coding regions (H). Table $\mathbf{S 4}$. Assembled haplotypes of mitochondrial electron transport chain contained sequences(H-ETC). Table S5. Assembled haplotypes of mitochondrial respiratory complex contained sequences (H-MRCl, H-MRCIV, and H-MRCV). Table S6. Assembled haplotypes of protein coding genes (H-ND4L, H-ND4, H-ND5, H-ND6, H-ATP6). Table S7. Assembled haplotypes of rRNA coding genes (H-12SrRNA, H-16SrRNA). Table S8. Statistics for the number of observations in each cell of the interaction between BMPR1B and T7719G in tRNA-Lys and corresponding effects on litter size. (XLSX $33 \mathrm{~kb}$ )

\section{Abbreviations}

mtDNA: Mitochondrial DNA; ETC: Electron transport chain; MRC: Mitochondrial respiratory complex; $\mathrm{H}$ : The haplotype constituted by all non-synonymous mutations in mitochondrial coding sequences; $\mathrm{HG}$ : The haplogroup classified by $\mathrm{H}$; H-ETC: The assembled haplotypes of mtDNA electron transport chain contained sequences; H-MRC: The assembled haplotypes of mtDNA mitochondrial respiratory complex contained sequences, including $\mathrm{H}-\mathrm{MRCl}$,

H-MRCIII, H-MRCIV, and H-MRCV, corresponding to complex I, III, IV and V, respectively; $\mathrm{H}$-gene: The assembled haplotypes of mitochondrial genes, including polypeptide-coding genes, rRNA genes and tRNA genes

\section{Acknowledgements}

The authors thank J. C. Jiang (China Agricultural University) for data analysis. The authors also thank Dr. J. M. Zhong and Ms. D. Miller (Auburn University) for language editing.

\section{Funding}

The research was supported by the Project of Science and Technology of Hebei Province (15226308D) and the National Key Basic Research Program of China (2014CB138500). The former collected the sample and fed the experimental sheep, and the latter designed the study, performed the experiments, analyzed the data, interpreted the data, and drafted the manuscript. 


\section{Availability of data and materials}

All data generated or analysed during this study are included in this published article and its supplementary information files.

\section{Authors' contributions}

XZ designed the study. WD and XC collected the sample and fed the experimental sheep. the $X C, H X, D B$ and TY performed the experiments. DW $\mathrm{HX}$ and $\mathrm{XC}$ analyzed the data. DW, HX, XC and XZ interpreted the data and drafted the manuscript. All authors read and approved the final manuscript.

\section{Competing interests}

The authors declare that they have no competing interests.

\section{Consent for publication}

Not applicable.

\section{Ethics approval}

All experimental sheep were maintained under the standard farm management practices according to the guidelines of the experimental animal management of China Agricultural University (CAU), and the study was approved by the Experimental Animal Care and Use Committee of CAU. Blood samples were collected followed the guideline of Animal Ethical Committee of China.

\section{Author details}

'College of Animal Science and Technology, China Agricultural University, Beijing 100193, China. ${ }^{2}$ Institute of Animal Science and Veterinary of Hebei Province, Baoding 071000, China.

Received: 7 September 2016 Accepted: 17 March 2017

Published online: 01 April 2017

\section{References}

1. Anderson S, Bankier AT, Barrell BG, de Bruijn MH, Coulson AR, Drouin J, et al. Sequence and organization of the human mitochondrial genome. Nature. 1981:290:457-65.

2. Bell BR, Mcdaniel BT, Robison OW. Effects of cytoplasmic inheritance on production traits of dairy-cattle. J Dairy Sci. 1985;68:2038-51.

3. Yen NT, Lin CS, Ju CC, Wang SC, Huang MC. Mitochondrial DNA polymorphism and determination of effects on reproductive trait in pigs. Reprod Domest Anim. 2007:42:387-92.

4. Fernandez Al, Alves E, Fernandez A, de Pedro E, Lopez-Garcia MA, Ovilo C, et al. Mitochondrial genome polymorphisms associated with longissimus muscle composition in Iberian pigs. J Anim Sci. 2008;86:1283-90.

5. Qin YH, Chen SY, Lai SJ. Polymorphisms of mitochondrial Atpase 8/6 genes and association with milk production traits in Holstein cows. Anim Biotechnol. 2012:23:204-12.

6. Paneto JCC, Ferraz JBS, Balieiro JCC, Bittar JFF, Ferreira MBD, Leite MB, et al. Bos indicus or Bos taurus mitochondrial DNA: comparison of productive and reproductive breeding values in a Guzerat dairy herd. Genet Mol Res. 2008;7:592-602.

7. Zhang B, Chen H, Hua LS, Zhang CL, Kang XT, Wang XZ, et al. Novel SNPs of the mtDNA ND5 gene and their associations with several growth traits in the Nanyang cattle breed. Biochem Genet. 2008;46:362-8.

8. Auricélio AM, Bittar JFF, Bassi PB, Ronda JB, Bittar ER, Panetto JCC, et al. Influence of endogamy and mitochondrial DNA on immunological parameters in cattle. BMC Vet Res. 2014;10:79-88.

9. Henry BA, Loughnan R, Hickford J, Young IR, St. John JC, Clarke I. Differences in mitochondrial DNA inheritance and function align with body conformation in genetically lean and fat sheep. J Anim Sci. 2015;93:2083-93.

10. Reicher S, Seroussi E, Weller Jl, Rosov A, Gootwine E. Ovine mitochondrial DNA sequence variation and its association with production and reproduction traits within an Afec-Assaf flock. J Anim Sci. 2012:90:2084-91.

11. Li S, Zadworny D, Aggrey SE, Kuhnlein U. Mitochondrial PEPCK: a highly polymorphic gene with alleles co-selected with Marek's disease resistance in chickens. Anim Genet. 1998;29:395-7.

12. Lu WW, Hou LL, Zhang WW, Zhang PF, Chen W, Kang X, et al. Study on heteroplasmic variation and the effect of chicken mitochondrial ND2. Mitochondrial DNA Part A, DNA mapping, sequencing, and analysis. 2016;27:2303-9.
13. Mulsant $P$, Lecerf $F$, Fabre $S$, Schibler L, Monget $P$, Lanneluc I, et al. Mutation in bone morphogenetic protein receptor-IB is associated with increased ovulation rate in Booroola Merino ewes. Proc Natl Acad Sci U S A. 2001:98:5104-9.

14. Hanford KJ, Snowder GD, Vleck LDV. Models with nuclear, cytoplasmic, and environmental effects for production traits of Columbia sheep. J Anim Sci. 2003:81:1926-32.

15. Van Vleck LD, Snowder GD, Hanford KJ. Models with cytoplasmic effects for birth, weaning, and fleece weights, and litter size, at birth for a population of Targhee sheep. J Anim Sci. 2003;81:61-7.

16. Snowder GD, Hanford KJ, Van Vleck LD. Comparison of models including cytoplasmic effects for traits of Rambouillet sheep. Livest Prod Sci. 2004;90:159-66.

17. Van Vleck LD, Hanford KJ, Snowder GD. Lack of evidence for cytoplasmic effects for four traits of Polypay sheep. J Anim Sci. 2005:83:552-6.

18. Sambrook J, Russell D. Molecular Cloning: A Laboratory Manual. 3rd ed. Cold Spring Harbor, New York: Cold Spring Harbor Laboratory Press; 2001.

19. Liu FL, Liu YB, Wang F, Wang R, Tian CY, Liu MX. Study on the polymorphism of Bone Morphogenetic Protein Receptor IB in China partial sheep. Acta Agr Boreali-Sinica. 2007;22:151-4.

20. Meadows JRS, Hiendleder S, Kijas JW. Haplogroup relationships between domestic and wild sheep resolved using a mitogenome panel. Heredity. 2011;106:700-6

21. Villesen P. FaBox: an online toolbox for fasta sequences. Mol Ecol Notes. 2007;7:965-8

22. Network 4.6.1.4. http://www.fluxus-engineering com. Accessed 5 Sept 2016.

23. R Core Team. R: A language and environment for statistical computing. Vienna: R Foundation for Statistical Computing; 2013. URL http://www.Rproject.org/.

24. Taylor RW, Turnbull DM. Mitochondrial DNA mutations in human disease. Nat Rev Genet. 2005:6:389-402

25. Haapalainen $E$, Laurinen $P$, Roning J, Kinnunen $H$. Estimation of exercise energy expenditure using a Wrist-Worn accelerometer: a linear mixed model approach with fixed-effect variable selection. Seventh International Conference on Machine Learning and Applications, Proceedings. 2008. p. 796-801.

26. A Human Mitochondrial Genome Database. http://www.mitomap.org. Accessed 4 May 2012

27. Wang $M$, Peng $Y$, Zheng J, Zheng $B$, Jin $X$, Liu $H$, et al. A deafnessassociated tRNAAsp mutation alters the m1G37 modification, aminoacylation and stability of tRNAAsp and mitochondrial function. Nucleic Acids Res. 2016:44:10974-10985.

28. Chen X, Zhang Y, Xu B, Cai Z, Wang L, Tian J, et al. The mitochondrial calcium uniporter is involved in mitochondrial calcium cycle dysfunction: Underlying mechanism of hypertension associated with mitochondrial tRNA(lle) A4263G mutation. Int J Biochem Cell Biol. 2016:78:307-14.

29. Li G, Duan YX, Zhang XB, Wu F. Mitochondrial tRNA mutations may be infrequent in hepatocellular carcinoma patients. Genet Mol Res. 2016;15.

\section{Submit your next manuscript to BioMed Central and we will help you at every step:}

- We accept pre-submission inquiries

- Our selector tool helps you to find the most relevant journal

- We provide round the clock customer support

- Convenient online submission

- Thorough peer review

- Inclusion in PubMed and all major indexing services

- Maximum visibility for your research

Submit your manuscript at www.biomedcentral.com/submit 